\title{
TIDAL CAPTURE IN STAR CLUSTERS
}

\author{
ROSEMARY A. MARDLING \\ Mathematics Department, \\ Monash University, Melbourne, Australia
}

\begin{abstract}
We review the tidal capture process and in particular the chaotic orbital evolution which follows capture. We discuss the formation of lowmass X-ray binaries in globular clusters via tidal capture and speculate on the possibility that some field low-mass X-ray binaries were formed this way in open clusters which have since dispersed, or in existing old open clusters which are not accessible to observation because of obscuration by dust or because they are indistinguishable from the rich background of galactic stars.
\end{abstract}

\section{Introduction}

Tidal capture is a mechanism for binary formation which involves transferring the excess energy of unbound orbital motion to the tides of two stars which pass each other at a distance of a few stellar radii so that a bound system can result. The process was originally suggested by Fabian, Pringle \& Rees (1975) to explain the origin of the X-ray sources being observed for the first time in globular clusters. It was suggested that these sources were neutron stars accreting from low-mass main sequence companions, ie., low-mass X-ray binaries (LMXBs). The idea appears to explain the excess of such systems. Since the X-ray luminosity to mass ratio is at least 100 times higher in globular clusters than in the galactic disk (Katz 1975), it is natural to assume that a process peculiar to globular clusters is responsible. The high stellar densities and low velocity dispersions found in globular clusters greatly favour the tidal capture mechanism, but it should be noted that open clusters have velocity dispersions an order of magnitude lower than globular clusters, so that although the stellar densities are also much lower, rare tidal captures may take place frequently enough to explain some 
of the field sources observed. This will be discussed in Section 3.1.

Considerable doubt has been cast recently on the ability of the tidal capture process to produce long-lived binaries, that is, binaries which avoid tidal disruption or self-ionization. Such conclusions have been drawn from heuristic discussions of the evolution following capture (with careful attention to energy deposition - McMillan, McDermott \& Taam 1987, Ray, Kembhavi \& Antia 1987), or studies of the dynamical evolution of extremely close binaries for at most a few orbits after capture (see, for example, Gingold \& Monaghan 1980, Rasio \& Shapiro 1991, Benz \& Hills 1992, Kochanek 1992). Such studies have been governed by the fact that in order to follow the evolution numerically after capture, the timestep is restricted by the dynamical timescales of the stars. For a typical capture, a star may oscillate more than a million times per orbit, at least for the first few orbits following capture, and this has severely limited the number of orbits accessible to study.

Recent work by the author (Mardling 1995a,b) has overcome this problem. By employing a normal mode analysis devised by Gingold \& Monaghan (1980), and taking advantage of the fact that the orbit and the tides interact for only part of a very long period orbit, one is able to study thousands of orbits following capture. It is found that for a short time, the orbital evolution is chaotic (large tides accompanied by unpredictable large changes in eccentricity) and after this phase (given that the binary survives) the orbital evolution becomes "normal" in that tides are small and the binary circularizes slowly. We will elaborate on this process in Section 2. Section 3 discusses the formation of LMXBs via tidal capture, including field objects. Section 4 briefly discusses the role of tidal capture binaries in cluster dynamics.

\section{Tidal Capture}

In this section we summarize the main elements of the tidal capture mechanism. A more extensive review of this subject may be found in Mardling (1996a).

\subsection{STELLAR SEPARATIONS FOR CAPTURE}

Tidal capture becomes possible when two stars pass each other so closely that their tides are able to absorb the excess energy of unbound orbital motion (Figure 1). For typical globular cluster velocity dispersions $(\sim$ $10 \mathrm{~km} \mathrm{~s}^{-1}$ ), this means passing within about 3 stellar radii for equal mass 


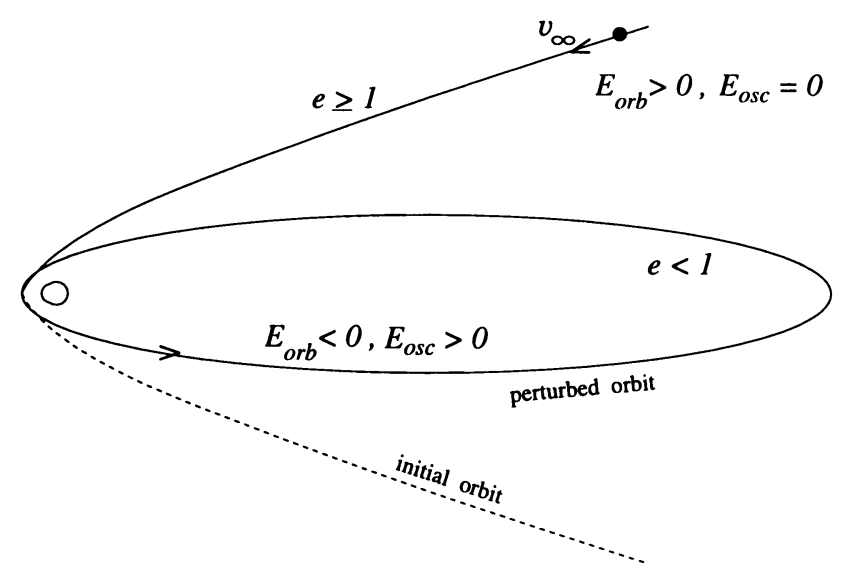

Figure 1. Tidal capture formation of a binary. $E_{\text {orb }}$ and $E_{\text {osc }}$ are the orbital binding energy and the tidal or oscillation energy respectively, and $e$ is the orbital eccentricity.

stars where one is compact. ${ }^{1}$ This distance increases with increasing mass ratio of compact to extended star, as well as with decreasing relative velocity at infinity (less energy to absorb). The process is enhanced by gravitational focussing, for example, the impact parameter for the equal mass system described above is about $1 \mathrm{AU}$. The situation is quite different in galactic nuclei where the velocity dispersions are much higher. ${ }^{2}$ In this case, gravitational focussing is ineffective and stars need to collide in order to absorb all the energy of unbound motion.

\subsection{CAPTURE RATES}

Given two species of stars with number densities $N_{1}$ and $N_{2}$, masses $M_{1}$ and $M_{2}$ living in an environment where the velocity dispersion is $\sigma$, the capture rate between them is given by (see, for example, Mardling 1996a)

$$
\Gamma_{12}=6.8 \frac{N_{1}}{10^{4} \mathrm{pc}^{-3}} \frac{N_{2}}{10^{4} \mathrm{pc}^{-3}} \frac{M_{1}+M_{2}}{M_{\odot}} \frac{R_{p}^{\min }}{R_{\odot}} \frac{10 \mathrm{~km} \mathrm{~s}^{-1}}{\sigma} \mathrm{Gyr}^{-1} \mathrm{pc}^{-3} .
$$

It should be noted that this formula includes collisions; in practise the rate at which long-lived binaries are formed by tidal capture will be a fraction of $\Gamma_{12}(\sim 25 \%$ ?). We apply this formula to capture of neutron stars in Section 3.1.

${ }^{1}$ This maximum periastron separation is for stars with convective envelopes; stars with radiative envelopes must pass even closer.

${ }^{2}$ The core of the nucleus of M33 is an exception; here the velocity dispersion is estimated to be as low as $21 \mathrm{~km} \mathrm{~s}^{-1}$ (Kormendy \& McClure 1991). 


\subsection{CHAOTIC ORBITAL EVOLUTION}

If a Lagrangian is employed to derive the equations of motion for a system, one gains the advantage of a conserved energy. In order to examine the tide-orbit coupling in a binary system, a suitable Lagrangian consists of terms describing the orbit, terms describing the fluid motion of the stars, and a term describing the interaction between the two. Gingold \& Monaghan (1980) contructed such a Lagrangian and used the normal modes of a polytrope and their associated orthogonality conditions to derive a Lagrangian which depends only on the orbital variables and the mode amplitudes (which describe the tides). The equations of motion derived from this consist of a Kepler orbit perturbed by the tides, and forced simple harmonic oscillators representing the orbital forcing of the tides. This system of equations is constrained by an energy and an angular momentum integral and it is this which makes the system self-consistent and allows one to examine in detail the tide-orbit interaction over many orbits.

For most binary orbits specified by the mass ratio, the orbital eccentricity and, say, the orbital period or the semi-major axis or the periastron separation, the solutions to these equations are periodic in that energy is exchanged between the tides and the orbit over a few (or sometimes many) orbits. Except for resonant orbits, that is, for orbits where the orbital period is nearly an integral number of oscillation periods of the most energetic mode, the tides remain small for periodic orbits. In contrast to this regular kind of behaviour, there exists a range of eccentricities and periastron separations for which the solutions are chaotic, that is, they exhibit extreme sensitivity to initial conditions. In addition, the orbit can (at least partially) circularize in the absence of dissipation, and this implies large tides.

Chaotic orbits are highly eccentric and generally extremely close at periastron. Capture orbits are clearly candidates for chaotic behaviour and in fact, one can show that all capture orbits are chaotic (Mardling, 1996b).

\subsection{DISSIPATION AND LONG-TERM EVOLUTION}

The tides raised during the chaotic phase following capture are generally extremely energetic, and it follows that nonlinear dissipative processes will be important. These in turn will limit the tidal energy, although the extent to which this is so is a matter of debate (Mardling 1995b, Kumar \& Goodman 1996). The response of the stars to the huge tidal energies has also generated much discussion. In general, it is necessary to know where in the star the tidal energy is deposited. If it is in the extreme outer layers or in the core, the system will not be much affected, while if it is somewhere in between, the star may expand and cause the system to enter a common envelope phase, possibly destroying the binary (Podsiadlowski 1996). 


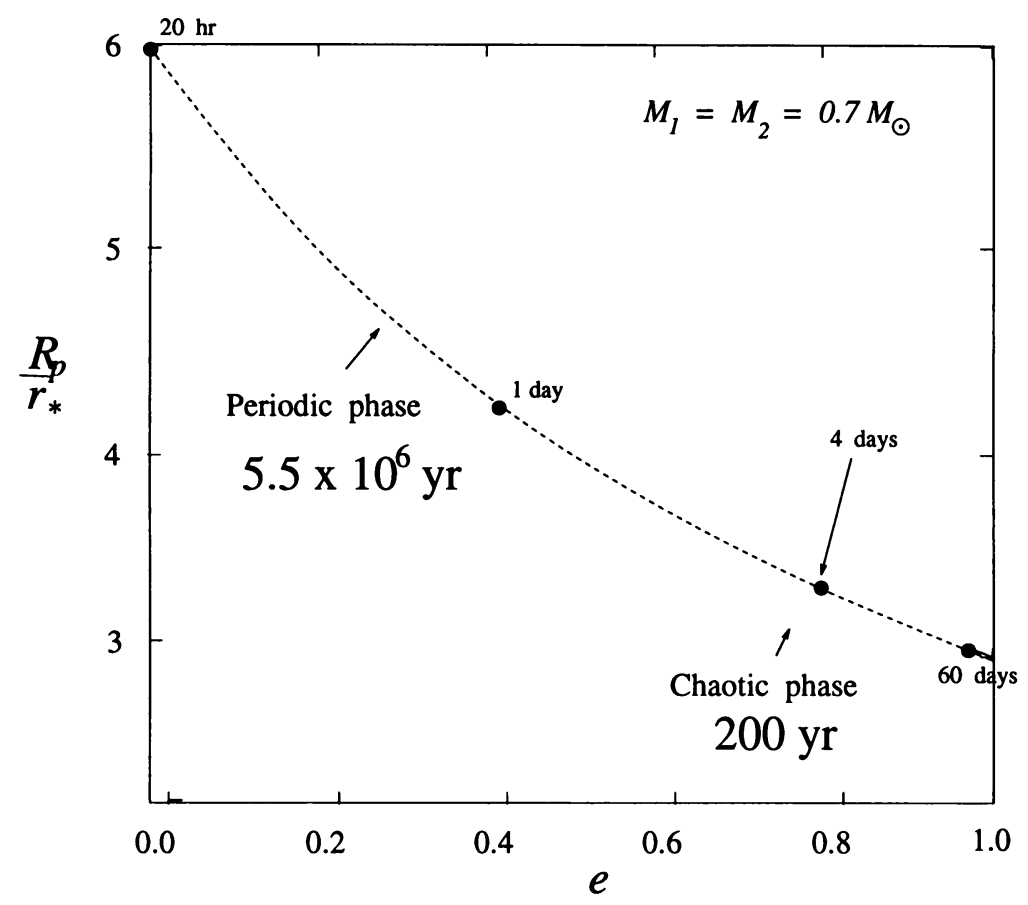

Figure 2. Chaotic and periodic circularization of an equal mass binary captured at 3 stellar radii. The times shown in days are the orbital periods at these points. The dashed curve represents constant orbital angular momentum.

Given that the system survives the short violent chaotic phase, it will eventually become quiescent in the sense of normal tidal circularization. This involves the system permanently crossing the boundary between chaotic and periodic behaviour after it has dissipated an amount of energy associated with the initial conditions at capture. For example, an equal mass system with an initial periastron separation of 3 stellar radii will leave the chaotic phase with an eccentricity of 0.8 , and will take at least another few million years to circularize, depending on the linear damping timescale. This is in contrast to previous estimates of the circularization time which were of the order of a few years (McMillan, McDermott \& Taam 1987).

Figure 2 illustrates the two phase circularization process by plotting the periastron separation against eccentricity for such a system. In this case, the nonlinear damping timescale is about $30 \mathrm{yr}$ and the linear damping timescale is $1000 \mathrm{yr}$. The nonlinear damping timescale may well be shorter than this, but whenever it is longer than the orbital period, chaotic behaviour will persist. In this case, the chaotic phase lasts for about 200 
years after which the binary takes more than 5 million years to finish circularizing.

\section{Low-Mass X-Ray Binaries via Tidal Capture}

The number of LMXBs per unit mass in our globular cluster system is at least two orders of magnitude higher than in the galaxy generally. This naturally leads to the suggestion that a process peculiar to globular clusters is responsible for this population of LMXBs, and that the field population is formed some other way. Fabian, Pringle \& Rees (1975) proposed tidal capture as a viable mechanism in globular clusters, while Hut, Murphy \& Verbunt (1991), faced with the suggestion that tidal capture binaries do not survive the circularization process (McMillan, McDermott \& Taam 1987, Ray, Kembhavi \& Antia 1987), suggested that globular cluster LMXBs are formed via exchange of neutron stars into primordial binaries. Mardling (1995b) has suggested that the exchange process generally does not produce binaries with periods as short as those observed. ${ }^{3}$ For example for solar mass stars, the cross sections for collisions and non-collisions are about equal for binaries with periods around 20 days, with collisions dominating for smaller period binaries (McMillan 1995) and clean exchanges tending to leave binaries with around the same period as the original binary. Since the companion in a neutron star binary thus formed will have a mass at or below the turn-off mass of the cluster, the mass ratio of the binary will be such that mass transfer will be stable once the secondary evolves. Thus this scenario cannot rely on a common envelope phase to shrink the binary. On the other hand, one might expect to observe some long period globular cluster LMXBs with giant components; there is no evidence for this so far. Magnetic breaking and gravitational radiation will also be ineffective for such orbital periods (at least for low to moderate eccentricities).

Some of these binaries will become harder through flyby interactions with other cluster stars (Davies 1995), but it is not clear whether such a process is capable of producing all the LMXBs observed. In addition, if LMXBs are the progenitors of millisecond pulsars, the process may need to be even more efficient.

It should be pointed out that in spite of the discussion above, it is still possible for this and other dynamical interactions (such as binary-binary interactions) to produce short period mass transfer binaries.

If the tidal capture process is viable, it should be capable of producing LMXBs directly. For example, if a turn-off mass $0.8 M_{\odot}$ main sequence star

\footnotetext{
${ }^{3}$ Although at present only three globular cluster LMXBs have known orbital periods, there is evidence to suggest that the other LMXBs also have short periods (Verbunt 1995).
} 
captures a neutron star at, say, 3.5 stellar radii, it will leave the chaotic phase with an eccentricity of around 0.9 and slowly circularize to a separation of 7 stellar radii. This is assuming there is no mass loss during the evolution, an assumption which almost certainly does not hold during the chaotic phase. With mass loss the orbit will widen at periastron and this may in fact reduce the length and intensity of the chaotic phase, although as the mass ratio of the system changes, so does the chaos boundary in a way which competes with the increasing orbital separation. On the other hand, the tides can absorb a significant fraction of the orbital angular momentum during the chaotic phase (the amount being proportional to the tidal energy) so that substantial mass loss can also mean substantial angular momentum loss. Throughout the whole circularization process, tidal forces will act to spin up the star. Magnetic breaking, effective in the low-mass main sequence stars of interest, will act against this spin-up so that orbital angular momentum will be continually lost during circularization. Note that old low-mass main sequence stars in globular clusters have negligible spin, precisely because of magnetic breaking, so that the spin-up process after capture could result in significant angular momentum loss from the system by the time of circularization (recall that the circularization time predicted by the model discussed in this paper is at least four orders of magnitude longer than previous estimates).

In the absence of angular momentum loss the binary described above, once circularized, will have an orbital period of around 24 hours. In order for mass tranfer to start, the secondary will either have to evolve (in this case to a radius of about $1.8 R_{\odot}$ ) or the orbit will have to shrink via magnetic breaking (or both). Stars less massive than the turn-off mass of the cluster must rely on the latter process. Of the three globular cluster LMXBs with known orbital periods, two are tidal capture candidates. The object in M15 which has an orbital period of 17.1 hours (Ilovaisky et al. 1993) must contain a slightly post main sequence donor, while the object in NGC 6441 with an orbital period of 5.7 hours (Sansom et al. 1993) must contain a main sequence star and may have started mass transfer after a period of orbital shrinkage via magnetic breaking (enhanced in the later stages by gravitational radiation).

\subsection{THE FIELD POPULATION OF LMXBS}

The main formation scenario for the field population involves common envelope evolution (van den Heuvel 1983), and there are many evolutionary contraints which a binary must satisfy in order to evolve to an LMXB via this channel. On the other hand, these objects are rare so that any model for their formation must necessarily reflect this. Webbink \& Kalogera (1994) 
table 1. Capture Rates for Neutron Stars

\begin{tabular}{rrrlcccc}
\hline & \multicolumn{1}{c}{$N_{1}$} & $N_{2}$ & \multicolumn{1}{c}{$M_{1} / M_{\odot}$} & $M_{2} / M_{\odot}$ & $R_{p} / R_{*}$ & $\sigma\left(\mathrm{km} \mathrm{s}^{-1}\right)$ & $\Gamma_{12}$ \\
\hline (a) & 30,000 & 900 & $0.8(\mathrm{MS})$ & 1.4 & 4 & 10 & 13 \\
$(b)$ & 300 & 3 & $1.0(\mathrm{MS})$ & 1.4 & 5 & 1 & 0.007 \\
$(c)$ & 100 & 3 & 1.0 (giant) & 1.4 & $2(?)$ & 1 & 0.01 \\
\hline
\end{tabular}

list nine constraints for LMXB formation via common envelope evolution and there are many uncertainties involved in each one, making statistical estimates of the number of LMXBs formed via this process difficult.

Here we propose that at least some of the field LMXBs were produced via tidal capture in open clusters which have since dispersed, or in existing old open clusters which are not accessible to observation because of obscuration by dust or which are indistinguishable from the rich background of galactic stars. An obvious problem with this scenario is cluster retention of neutron stars. We appeal to the fact that globular clusters manage to retain a significant number of neutron stars as evidenced by their large population of LMXBs and millisecond pulsars, despite having escape velocities of only a few tens of kilometers per second. While the escape velocities of open clusters are lower still, the process which allows globular clusters to retain some neutron stars might be expected to operate in open clusters as well.

Table 1 uses equation 1 to estimate the capture rates of neutron stars for various hypothetical clusters, where $N_{2}$ is the number density of neutron stars. Case (a) gives the capture rate of neutron stars by turn-off mass main sequence stars in a globular cluster like 47 Tuc; these numbers are taken from Davies \& Benz (1995). Case (b) might represent an old open cluster containing 10,000 stars, one which is two orders of magnitude less massive than 47 Tuc. We take $N_{1}$ to be simply a hundredth of the corresponding value for case $(a)$. Since the neutron star content of such a cluster is unknown, we take $N_{2}$ to be a third of a hundredth of the corresponding value for case $(a)$. Case $(c)$ considers capture of neutron stars by giants of radius $\sim 50 R_{\odot}$ in the same open cluster as case $(b)$. While these numbers are highly speculative, the results serve to illustrate that given such neutron star densities, tidal capture of neutron stars in open clusters must be considered seriously. For instance, although the capture rate for case (b) is low, this represents 7 captures per 1000 clusters per Gyr, and given the large number of such clusters which exist in the galaxy over a Gyr, as well as the low birthrate and longevity of LMXBs, it is not unreasonable 
to suggest that some LMXBs are produced in this way.

$N$-body simulations of open clusters containing 10,000 stars (Aarseth, this volume) have revealed several two-body hyperbolic encounters with closest approach separations less than a few stellar radii. None resulted in tidal capture because in each case the relative velocity was too high. Nonetheless, given that only a few such calculations have been performed to date, these results lend some support to the above estimate for capture rates in open clusters.

\subsection{CATACLYSMIC VARIABLES}

There has been considerable debate in recent years about the existence or otherwise of cataclysmic variables (CVs) in globular clusters. Grindlay \& Cool (this volume) argue that the abundant soft X-ray sources are CVs, while Shara (this volume) argues that more should be observed in outburst if these sources are indeed CVs. One might expect that if tidal capture is efficient at making LMXBs, then it should be efficient at making CVs. The tidal capture process is still not well understood, particularly the stellar response to the chaotic phase, and it may well be that a better understanding will lead to a resolution of this problem.

\section{Tidal Capture Binaries and Cluster Dynamics}

Before the discovery of giants in the outer regions of some globular clusters (Pryor et al. 1989), it was believed that such clusters were devoid of primordial binaries (Gunn \& Griffin 1979). At the same time, it was known that binaries are necessary for core support against collapse and for reexpansion of the core, and tidal capture binaries were shown to be capable of performing these roles (Statler, Ostriker \& Cohn 1987). Assumed to be extremely hard circular binaries, they were found to be an indirect heat source in that they tended to be ejected from the core.

More recent calculations have included the effect of primordial binaries on cluster evolution (McMillan, Hut \& Makino 1990, 1991 and Heggie $\&$ Aarseth 1992) and these are found to be a much more efficient direct energy source for the core. $N$-body calculations now include more realistic circularization times for tidal capture binaries (Aarseth, this volume, Mardling \& Aarseth, 1996), but it will be necessary to wait until larger values of $N$ are achieved before the role of tidal capture binaries in globular cluster dynamics becomes apparent (note that the circularization times for such binaries are comparable to the central relaxation timescales for many globular clusters). 


\section{Acknowledgements}

I thank Dr Jun Makino and Professor Daiichiro Sugimoto for inviting me to Tokyo, and my colleagues and friends Drs Sverre Aarseth, Fred Rasio, Steve McMillan and Piet Hut for getting me there. Thanks also to Dr Frank Verbunt for introducing me to Noh theater.

\section{References}

Brandt, N. and Podsiadlowski, Ph.: 1995, Mon. Not. R. Astron. Soc. , 274, 461

Davies, M. B. : 1995, Mon. Not. R. Astron. Soc., in press

Davies, M. B. and Benz, W.: 1995, Mon. Not. R. Astron. Soc., in press

Fabian, A. C., Pringle, J. E. and Rees, M. J.: 1975, Mon. Not. R. Astron. Soc., 172, 15P Gingold, R. A. and Monaghan, J. J.: 1980, Mon. Not. R. Astron. Soc. , 191, 897

Gunn, J. E. and Griffin, R. F.: 1979, Astronom. J., 84, 752

Heggie, D. C. and Aarseth, S. J.: 1992, Mon. Not. R. astr. Soc. , 257, 513

Hut, P., Murphy, B. W. and Verbunt, F.: 1991, Astron. \& Astrophys., 241, 137

Ilovaisky, S. A., Aurière, M., Koch-Miramond, L., Chevalier, C., Cordoni, J.-P. \& Crowe, R. A. 1993, Astron. \& Astrophys., 270, 139

Katz, J. I.: 1975, Nature, 253, 698

Kormendy, J. and McClure, R. D.: 1993, Astronom. J. , 105, 1793

Kumar, P. and Goodman, J.: 1996, submitted

Mardling, R. A.: 1995a, Astrophys. J., 450, 722

Mardling, R. A.: 1995b, Astrophys. J. , 450, 732

Mardling, R. A.: 1996a, to appear in Evolutionary Processes in Binary Stars, eds R. A. M. J. Wijers, M. B. Davies and C. A. Tout, Kluwer, Dordrecht, Holland

Mardling, R. A.: 1996b, in preparation

Mardling, R. A. and Aarseth, S. J.: 1996, in preparation

McMillan, S. L. W., 1995, private communication

McMillan, S. L. W., Hut, P. and Makino, J.: 1990, Astrophys. J. , 362, 522

McMillan, S. L. W., Hut, P. and Makino, J.: 1991, Astrophys. J. , 372, 111

McMillan, S. L. W., McDermott, P. N. and Taam, R. E.: 1987, Astrophys. J. , 318, 261

Podsiadlowski, Ph.: 1996, Mon. Not. R. Astron. Soc., in press

Pryor, C., McClure, R. D., Hesser, J. E. and Fletcher, J. M.: 1989, in Dynamics of Dense Stellar Systems, ed. D. Merritt, (New York: Cambridge University Press), 175

Ray, A., Kembhavi, A. K. and Antia, H. M.: 1987, Astron. \& Astrophys. 184, 164

Sansom, A. E., Dotani, T., Asai, K. \& Lehto, H. J. 1993, Mon. Not. R. astr. Soc. , 262, 429

Statler, T. S., Ostriker, J. P. and Cohn, H. N.: 1987, Astrophys. J. , 316, 626

van den Heuvel, E. P. J.: 1983, in Accretion-driven Stellar X-ray Sources, eds W. H. G. Lewin and E. P. J. van den Heuvel, Cambridge University Press, p. 303

Verbunt, F.: 1995, private communication

Webbink, R. F. and Kalogera, V.: 1994, in Evolution of X-Ray Binaries, eds S. S. Holt and C. S. Day, AIP Press, New York, p 321 